\title{
Preface
}

\section{Management of the Anterior Cruciate Ligament Deficient Knee}

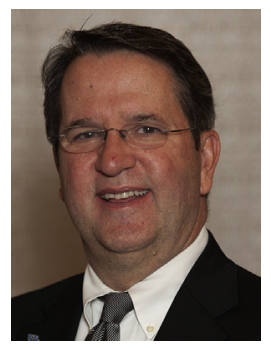

Darren L. Johnson, MD

Editor

I am very honored to be able to serve as the editor for this outstanding issue of Clinics in Sports Medicine on the management of the anterior cruciate ligament-deficient knee. I am confident that if you were to pool the editors-in-chief of our major orthopedic peer-reviewed journals, anterior cruciate ligament as a submission for publication would probably be the number one topic of submissions. Clearly, there is great interest in this topic given the number of anterior cruciate ligament disruptions that happen annually in this country. It seems that number is increasing each and every year. Over the last 30 years of orthopedic management of this complex problem, much has changed; much is currently being investigated, and we have much to learn moving forward. Over the last 10 years, there has been a renowned focus with respect to detailed anterior cruciate ligament anatomy as it pertains to our surgical reconstruction techniques. I think clearly that Dr Freddie Fu from the University of Pittsburgh was instrumental in getting all of us surgeons in North America to refocus on the detailed anatomy of the anterior cruciate ligament attachment sites. I clearly think and believe that this renowned focus on what Dr Freddie Fu has taught us has enabled us to become better anterior cruciate ligament surgeons. We should all be thankful to him for starting this discussion in this country over 10 years ago. However, there is much to be learned moving forward in improving our results of anterior cruciate ligament surgery. Having been an anterior cruciate ligament surgeon myself over the last 23 years, I am still humbled and perplexed by this unique problem that I see in the office on a weekly basis. Clearly all of my patients do not do well and not all of them return to anterior cruciate ligament-dependent sports at their previous level. For me personally, I have learned a great deal by the contributions of this distinguished group of authors. I am confident that I am a better physician in managing anterior cruciate ligament-deficient knee problems having read in great detail this outstanding issue. 
I have asked a special group of authors to contribute their knowledge and expertise to this issue of Clinics in Sports Medicine. As I am sure you'll testify, they have done a simply remarkable job. These authors have contributed much of their academic careers to what we know about anterior cruciate ligament injury and its management. They are on the cutting edge of making advancements in the treatment of this unique challenge, which we have not solved yet. I want to personally thank each and every contributor for donating their valuable time and energy to this educational effort. It is a true honor of mine to have worked with this special unique group in making this a tremendous success. I hope you enjoy this issue as much as I have enjoyed putting it together. I sincerely hope that it enables you to provide better care to the patients we are so lucky to have served. We as well as our patients will benefit tremendously.

Darren L. Johnson, MD Director of Sports Medicine Department of Orthopaedic Surgery University of Kentucky School of Medicine Lexington, KY 40536-0284, USA

E-mail address: 